\title{
Isolation and Properties of Strains of Micrococcus (Deinococcus) radiodurans Unable to Excise Ultraviolet Light-induced Pyrimidine Dimers from DNA: Evidence for Two Excision Pathways
}

\author{
By B. E. B. MOSELEY* AND DAVID M. EVANS \\ Department of Microbiology, University of Edinburgh, School of Agriculture, \\ Edinburgh EH9 3JG, Scotland, U.K.
}

(Received 19 January 1983)

\begin{abstract}
A mutant of Deinococcus (formerly Micrococcus) radiodurans (strain 302, mutant in $m t c A$ ) sensitive to both the lethal effect of mitomycin $\mathrm{C}$ and the mutagenic effect of simple alkylating agents, but having wild-type resistance to UV light, was treated with the mutagen $N$-methyl- $N^{\prime}$ nitro- $N$-nitrosoguanidine in an attempt to isolate strains deficient in the ability to excise UVinduced pyrimidine dimers. Three strains were isolated that were UV-sensitive, but had wildtype resistance to the lethal effect of methyl methanesulphonate and all were shown to be unable to excise pyrimidine dimers. The three strains UVS9, UVS25 and UVS78 had, in addition to the mutation in $m t c A$, mutations in loci designated $u v s C$, $u v s D$ and $u v s E$, respectively. When the mutant $m t c A$ gene was replaced by its wild-type allele in all three strains they became UV-and mitomycin C-resistant. On incubating the double mutants UVS9, UVS25 and UVS78 with wildtype DNA about $50 \%$ of the transformants selected for UV resistance were mitomycin Csensitive and about $50 \%$ resistant depending on whether the mutant $m t c A$ or the uvsC, $D$ or $E$ genes had been replaced by their wild-type alleles. Although strains mutant singly in uvs $C, D$ or $E$ were UV-resistant the rates of excision of pyrimidine dimers differed between them and was slower in all of them than in the wild-type and strain 302. The results indicate that wild-type $D$. radiodurans possesses two pathways for the excision of pyrimidine dimers and that mutational blocks in both must exist for the excisionless phenotype to be expressed.
\end{abstract}

\section{INTRODUCTION}

Deinococcus radiodurans (formerly Micrococcus radiodurans; Brooks \& Murray, 1981) is the type species of a small number of exceptionally radiation resistant, red-pigmented cocci considered to comprise one of the eight major groups of the Eubacteria (Fox et al., 1980). Only two mechanisms for the repair of UV light-damaged DNA in $D$. radiodurans have been described, namely excision and recombination repair (Boling \& Setlow, 1966; Moseley et al., 1972; Moseley \& Copland, 1975). However, in spite of the isolation of a number of UV-sensitive mutants of $D$. radiodurans from populations of the wild-type treated with the mutagen $N$-methyl$N^{\prime}$-nitro- $N$-nitrosoguanidine (MNNG) none has been defective in the excision of UV-induced pyrimidine dimers (Moseley, 1967, 1983; Moseley \& Copland, 1975, 1978), an unexpected result since such mutants were the first UV-sensitive strains of Escherichia coli to be isolated (Hill, 1958; Howard-Flanders \& Theriot, 1962). This lack of success in isolating excision-defective mutants suggested either that excision repair contributes so little to the overall repair capacity of D. radiodurans, compared with recombination repair, that the difference in UV-sensitivity

Abbreviations: MMS, methyl methanesulphonate; MNNG, $N$-methyl- $N^{\prime}$-nitro- $N$-nitrosoguanidine; MTC, mitomycin $\mathrm{C}$. 
between excision-deficient and excision-proficient strains is not sufficiently large to allow the mutant to be isolated, or that mutation to give the excisionless phenotype is a very rare event.

A mitomycin C (MTC)-sensitive, UV-resistant mutant of $D$. radiodurans (strain 302, mutant in $m t c A$ ) has been isolated (Moseley \& Copland, 1978) that is hypermutable by MNNG (Tempest \& Moseley, 1978, 1980). On the basis that mutation to the excisionless phenotype is a rare event, it was decided to increase the chance of isolating such mutants by treating strain 302 , rather than the wild-type, with MNNG. Having obtained putative excision-deficient mutants, i.e. displaying an UV-sensitive, methyl methanesulphonate (MMS)-resistant phenotype, the $m t c A$ mutation could then be removed by transforming the double mutant with DNA from a strain wild-type for $m t c A$ and selecting for MTC resistance (Moseley \& Copland, 1978).

Several UV-sensitive, excisionless strains were isolated. However, when the $m t c A$ gene in each was replaced by its wild-type allele all the mutants became UV-resistant. The resolution of this problem provides evidence for the existence of two, alternative, excision pathways in $D$. radiodurans.

\section{METHODS}

Bacteria. The strains of Deinococcus radiodurans used were all derivatives of D. radiodurans $\mathrm{R}_{1}$ (Anderson et al., 1956). They were: 302 , mutant in $m t c A$ and sensitive to MTC; 301, mutant in $m t c A$ and $u v s A$ and sensitive to MTC and UV radiation. Both 301 and 302 were sensitive on agar to $4 \mu \mathrm{g}$ rifampicin $\mathrm{ml}^{-1}$. Deinococcus radiodurans (Krase), resistant to $100 \mu \mathrm{g}$ rifampicin $\mathrm{ml}^{-1}$ was used as a source of transforming DNA (Tirgari \& Moseley, 1980).

Media. TGY medium for growth contained $5 \mathrm{~g}$ Bactotryptone (Difco), $1 \mathrm{~g}$ glucose and $3 \mathrm{~g}$ yeast extract (Difco) in 11 distilled water. TGY agar was made by solidifying this medium with $15 \mathrm{~g}$ Bactoagar $1^{-1}$. Phosphate buffer $(0.67 \mathrm{M}, \mathrm{pH} 7.0)$ for washing bacteria and suspending them for irradiation contained $4.73 \mathrm{~g} \mathrm{Na}_{2} \mathrm{HPO}_{4}$ and $4.54 \mathrm{~g}$ $\mathrm{KH}_{2} \mathrm{PO}_{4}$ in 11 distilled water.

Growth of bacteria. Exponential phase cultures were obtained by diluting samples from $18 \mathrm{~h}$ cultures into $20 \mathrm{ml}$ amounts of TGY medium, in $250 \mathrm{ml}$ conical flasks, to give a turbidity of about 0.08 in a nephelometer (EEL, Halstead, Essex) with an orange filter. The cultures were incubated with shaking at $30^{\circ} \mathrm{C}$ until they reached an appropriate turbidity. A turbidity of 0.30 was equivalent to $1.0 \times 10^{8}$ viable units $\mathrm{ml}^{-1}$.

Mutagenesis. To $10 \mathrm{ml}$ of an exponential phase culture of $D$. radiodurans 302 growing at $30^{\circ} \mathrm{C}$ and containing $1 \cdot 3$ $\times 10^{8}$ viable units $\mathrm{ml}^{-1}, 0.2 \mathrm{ml} \mathrm{MNNG}$ in phosphate buffer $\left(1 \mathrm{mg} \mathrm{ml}^{-1}\right)$ was added to give a final concentration of $20 \mu \mathrm{g} \mathrm{MNNG} \mathrm{ml} \mathrm{g}^{-1}$ and the culture was reincubated. At intervals up to $120 \mathrm{~min}$ two $0.1 \mathrm{ml}$ samples were removed, one diluted and plated on TGY agar to measure loss of viability, colonies from surviving bacteria being counted after 2 to $3 \mathrm{~d}$ at $30^{\circ} \mathrm{C}$, while the other sample was diluted into $20 \mathrm{ml} \mathrm{TGY}$ medium and incubated for $18 \mathrm{~h}$ at $30^{\circ} \mathrm{C}$ with shaking. This diluted the MNNG to below the MIC and consequent growth allowed recessive mutations to segregate.

Isolation of mutants. Samples from mutagenized cultures were diluted in TGY medium and $0.1 \mathrm{ml}$ amounts plated on TGY agar to give about 80 colonies per plate after incubation at $30^{\circ} \mathrm{C}$ for $2 \mathrm{~d}$. Two replicate copies of each plate were made on TGY agar, one copy being irradiated with a UV dose of $500 \mathrm{~J} \mathrm{~m}^{-2}$. Both copies were incubated at $30^{\circ} \mathrm{C}$ for $24 \mathrm{~h}$ and colonies that grew only on the unirradiated copy were tested individually for sensitivity to UV radiation. Initially, putative UV-sensitive clones were streaked in a paralleled array across the surfaces of TGY plates and increasing doses of UV radiation applied along the streaks. After incubation at $30^{\circ} \mathrm{C}$ for 1 to $2 \mathrm{~d}$ those clones more sensitive to $\mathrm{UV}$ radiation than strain 302 were clearly distinguishable and irradiation survival curves were obtained.

Irradiation survival curves. Bacteria from exponential phase cultures containing about $1.0 \times 10^{8}$ viable units $\mathrm{ml}^{-1}$ were centrifuged, washed and resuspended in phosphate buffer at the same concentration. For UV irradiation a $5 \mathrm{ml}$ sample of the washed suspension in a $9 \mathrm{~cm}$ Petri dish was irradiated at a distance of $40 \mathrm{~cm}$ from an Hanovia germicidal lamp, the suspension being agitated by a magnetic stirrer. The incident dose rate was $1.05 \mathrm{~J} \mathrm{~m}^{-2}$. Gamma irradiation was carried out in a ${ }^{60} \mathrm{Co}$ source at a dose rate of $3.0 \mathrm{krad} \mathrm{min}^{-1}$. Samples of the washed bacterial suspension $(3 \mathrm{ml})$ were irradiated, oxygen being bubbled through the suspension during irradiation.

At intervals $0 \cdot 1 \mathrm{ml}$ samples from the populations being UV or $\gamma$ irradiated were diluted in TGY medium and $0.1 \mathrm{ml}$ samples spread on TGY agar plates. Colonies were counted after incubation at $30^{\circ} \mathrm{C}$ for 3 to $4 \mathrm{~d}$.

Resistance to methyl methanesulphonate. The bacteria from $10 \mathrm{ml}$ amounts of exponentially growing cultures containing about $10^{8}$ viable units $\mathrm{ml}^{-1}$ were centrifuged, washed and resuspended in $10 \mathrm{ml}$ quantities of phosphate buffer and placed in $250 \mathrm{ml}$ conical flasks. MMS $(50 \mu \mathrm{l})$ was added to each flask and the suspensions incubated for $50 \mathrm{~min}$ at $30^{\circ} \mathrm{C}$. Appropriate dilutions of the suspension $(0.1 \mathrm{ml})$ were spread on TGY plates and colonies from surviving bacteria were counted after incubation at $30^{\circ} \mathrm{C}$ for 2 to $3 \mathrm{~d}$. 
Assay for sensitivity or resistance of cultures to alkylation mutagenesis. Wild-type $D$. radiodurans is very resistant to alkylation mutagenesis while strain 302 is very sensitive. The two cultures were used as controls in an assay for sensitivity or resistance to ethyl methanesulphonate (EMS) mutagenesis. One drop (approximately $0.02 \mathrm{ml}$ ) of EMS was added to one of two $0.8 \mathrm{ml}$ cultures in exponential phase growth, the second culture acting as a nontreated control. After $45 \mathrm{~min}$ incubation at $30^{\circ} \mathrm{C} 0 \cdot 1 \mathrm{ml}$ from each culture was added to $10 \mathrm{ml}$ amounts of sterile TGY in $250 \mathrm{ml}$ flasks and the cultures incubated for $18 \mathrm{~h}$. Samples of the undiluted cultures $(0 \cdot 1 \mathrm{ml})$ were spread on TGY agar plates containing $10 \mu \mathrm{g}$ rifampicin $\mathrm{ml}^{-1}$ to assay the number of rifampicin-resistant mutants and, when necessary, $0.1 \mathrm{ml}$ amounts of appropriately diluted culture on TGY agar plates to assay viable numbers. Colonies were counted after $3 \mathrm{~d}$ incubation at $30^{\circ} \mathrm{C}$. Using this protocol the frequency of rifampicin-resistant mutants in an EMS treated population of wild-type $D$. radiodurans was $2.0 \times 10^{-7}$ compared with a spontaneous frequency of $5.2 \times 10^{-8}$, i.e. a $3 \cdot 8$-fold increase. For strain 302 the EMS induced frequency was $1 \cdot 5 \times 10^{-4}$ compared with a spontaneous frequency of $1.0 \times 10^{-7}$, i.e. a 1500 -fold increase. In order to score clones as resistant or sensitive it was unnecessary to measure viable numbers if the cultures were turbid since resistant clones gave 10 to 50 colonies per $0 \cdot 1 \mathrm{ml}$ undiluted culture and sensitive clones gave 1000 to 5000 colonies on rifampicin agar.

To test for UV mutability. Exponential phase cultures were prepared for UV radiation by centrifugation and resuspension into phosphate buffer. A $0.1 \mathrm{ml}$ sample of unirradiated suspension was added to $10 \mathrm{ml}$ sterile TGY in a $250 \mathrm{ml}$ flask as an unirradiated control. A $5 \mathrm{ml}$ portion of the suspension was irradiated with a suitable dose of $\mathrm{UV}$ and $0.1 \mathrm{ml}$ amounts added to $10 \mathrm{ml}$ quantities of TGY in $250 \mathrm{ml}$ flasks. All the cultures were incubated for $48 \mathrm{~h}$ and $0 \cdot 1 \mathrm{ml}$ amounts of suitable dilutions were spread on TGY plates for viable counts and on TGY plates plus $10 \mu \mathrm{g}$ rifampicin $\mathrm{ml}^{-1}$ for counts of rifampicin-resistant mutants.

Preparation of transforming DNA and the transformation procedure. These have been described previously in detail (Moseley \& Copland, 1978). The selection of bacteria transformed from UV sensitivity to resistance was carried out using a dose of $540 \mathrm{~J} \mathrm{~m}^{-2}$ and of bacteria transformed from MTC sensitivity to resistance by plating on TGY agar containing MTC (Sigma) at a concentration of $0.05 \mu \mathrm{g} \mathrm{ml}^{-1}$.

Measurement of pyrimidine dimer excision from the DNA of UV irradiated D. radiodurans. A $25 \mu$ l sample of an $18 \mathrm{~h}$ culture of $D$. radiodurans and $125 \mu \mathrm{Ci}$ tritiated thymidine [specific activity $27 \mathrm{Ci} \mathrm{mmol}^{-1}\left(0.99 \mathrm{TBq} \mathrm{mmol}^{-1}\right)$, $1 \mathrm{mCi} \mathrm{ml}^{-1}\left(37 \mathrm{MBq} \mathrm{ml}^{-1}\right)$ ] were added to $2.5 \mathrm{ml} \mathrm{TGY}$ medium and incubated with shaking at $30^{\circ} \mathrm{C}$ for $18 \mathrm{~h}$. The labelled bacteria were centrifuged, resuspended in $5 \mathrm{ml} \mathrm{TGY}$ at $30^{\circ} \mathrm{C}$ and incubated for $1 \mathrm{~h}$ at $30^{\circ} \mathrm{C}$. The culture was centrifuged, the bacteria resuspended in $2.5 \mathrm{ml}$ phosphate buffer and UV irradiated with a dose of $360 \mathrm{~J} \mathrm{~m}^{-2}$. The irradiated suspensions were centrifuged, the bacteria resuspended in $2.5 \mathrm{ml} \mathrm{TGY}$ at $30^{\circ} \mathrm{C}$ and incubated in $0.5 \mathrm{ml}$ amounts in microcentrifuge tubes. At varying times excision was terminated by adding $0 \cdot 1 \mathrm{ml}$ calf thymus DNA ( $5 \mathrm{mg} \mathrm{ml}^{-1}$ ) and $0.6 \mathrm{ml}$ ice-cold $12.5 \% \mathrm{TCA}$ to each tube. The tubes were held on ice for $10 \mathrm{~min}$, centrifuged and the precipitate washed with ice-cold $5 \%$ TCA and then with ice-cold ethanol. The pellets obtained from centrifugation were dried at $50{ }^{\circ} \mathrm{C}$ and resuspended in $0.2 \mathrm{ml} 98 \%$ formic acid. The suspensions were transferred to $1 \mathrm{ml}$ freeze-drying ampoules which were then sealed and heated to $175^{\circ} \mathrm{C}$ for $1 \mathrm{~h}$. The brown hydrolysates were spotted on to Whatman $3 \mathrm{MM}$ chromatography paper, dried and run for $18 \mathrm{~h}$ in butanol/acetic acid/water (18:12:30, by vol.). The paper was dried, cut into $1 \mathrm{~cm}$ strips and each strip placed in a scintillation vial containing $0.5 \mathrm{ml}$ water to dissolve the labelled nucleotides. Scintillant $(4.5 \mathrm{ml})$ (NE 250, Nuclear Enterprises, Edinburgh) was added to each vial and the radioactivity assayed in a scintillation counter.

The percentage of thymine present in thymine-containing pyrimidine dimers was calculated from the ratio of counts formed in the dimer peak and the thymine peak.

\section{RESULTS}

\section{Isolation of putative excision deficient mutants}

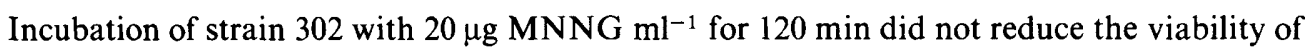
the culture. After allowing treated cells to segregate recessive mutations in liquid culture before plating, resulting colonies were replicated and the newly replicated plates UV irradiated. Of about 6000 colonies six were identified as sensitive to UV radiation after the second screening procedure. These were tested for their resistance to the lethal action of MMS, using wild-type $D$. radiodurans and strain 301 as controls for MMS resistance and sensitivity, respectively. The survival level of the wild-type after $50 \mathrm{~min}$ incubation in $5 \mu \mathrm{l}$ MMS per ml of bacterial suspension was $3.4 \times 10^{-1}$ and for 301 was $<7 \times 10^{-7}$. Three of the six mutants, strains UVS9, 25 and 78 , were resistant to MMS, having survival levels of $7.2 \times 10^{-1}, 7.7 \times 10^{-1}$ and $3.3 \times$ $10^{-1}$, respectively.

The UV and $\gamma$ radiation survival curves of these three strains are shown in Fig. 1 and an 

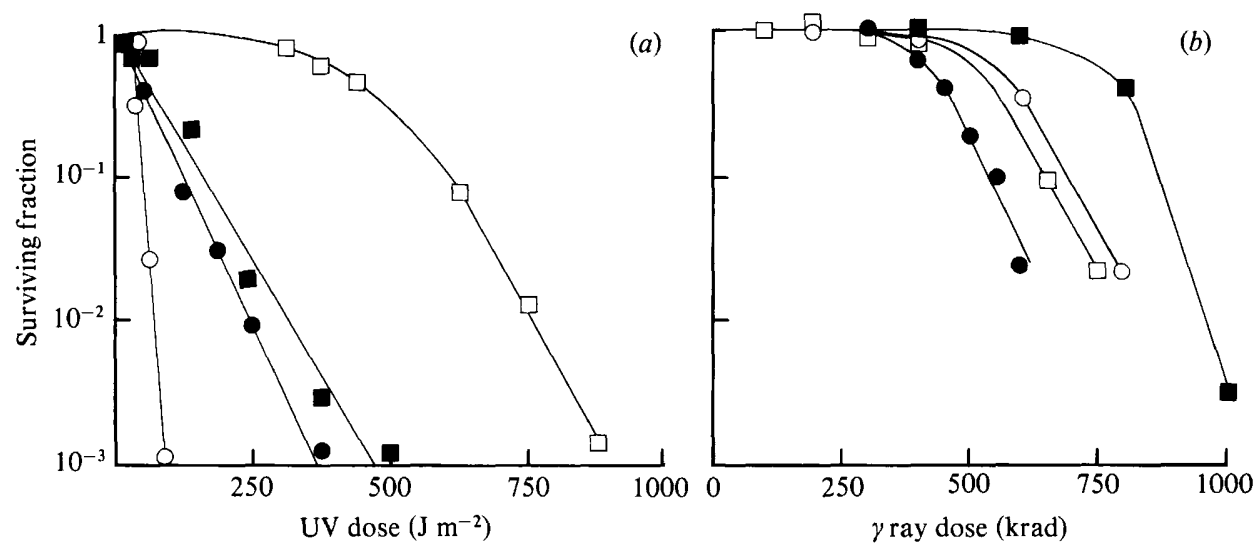

Fig. 1. (a) UV and (b) gamma irradiation survival curves of strains UVS9 (O), UVS25( $\square$ ) and UVS78 (O) compared with those of the wild-type strain of Deinococcus radiodurans $(\square)$.

Table 1. Analysis of survival curves of strains UVS9, UVS25 and UVS78 of D. radiodurans The results are derived from Fig. 1.

\begin{tabular}{|c|c|c|c|c|c|c|c|c|}
\hline \multirow[b]{2}{*}{ Strain } & \multicolumn{4}{|c|}{ UV radiation } & \multicolumn{4}{|c|}{ Ionizing radiation } \\
\hline & Intercept & $1 / \mathrm{e}$ & D37 & $\begin{array}{c}\text { Ratio D37 } \\
\text { wild-type } \\
\left(570 \mathrm{~J} \mathrm{~m}^{-2}\right) \\
\text { D37 mutant }\end{array}$ & Intercept & $1 / \mathrm{e}$ & D37 & $\begin{array}{c}\text { Ratio D37 } \\
\text { wild-type } \\
\text { (600 krad)/ } \\
\text { D37 mutant }\end{array}$ \\
\hline UVS9 & 0 & 54 & 54 & $10 \cdot 5$ & 375 & 25 & 400 & $1 \cdot 5$ \\
\hline UVS25 & 0 & 70 & 70 & 8 & 815 & 25 & 840 & $0 \cdot 7$ \\
\hline UVS78 & 33 & 8 & 40 & 14 & 695 & 25 & 585 & $1 \cdot 03$ \\
\hline
\end{tabular}

analysis of the curves in Table 1. Strains UVS9 and 25 have no shoulder to their UV survival curves and an exponential inactivation slope almost equal to that of the wild-type, i.e. the new mutations have increased the sensitivity of strain 302 causing complete loss of the shoulder. UVS78 has a small shoulder to its UV survival curve, but the exponential loss of viability is much more sensitive than that of the wild type. The $\gamma$-ray survival curves show UVS 25 to be more resistant, UVS78 to be as resistant and UVS9 to be slightly more sensitive, than the wildtype.

\section{Transformation to UV resistance of each strain with DNA from the others}

To show whether the new mutations introduced into strain 302 , to create the UV sensitive strains UVS9, 25 and 78, are in the same or different genes each of the strains was incubated with DNA from each of the strains and the wild-type made resistant to rifampicin and selection made independently for UV-resistant and rifampicin-resistant transformants. The results are shown in Table 2. Strains UVS78 and UVS9 gave much higher frequencies of transformation for both markers than UVS25, for reasons that have not been investigated. However, it is clear that UVS78 could be transformed to UV resistance equally well using DNA from the wild-type, UVS9 and UVS25 and that UVS9 could be transformed to UV resistance using DNA from UVS25 and UVS78, although not as well as by DNA from wild-type. These results indicate that the mutations creating the strains UVS9, UVS25 and UVS78 are in different genes and these were designated $u v s C$, uvs $D$ and $u v s E$, respectively.

\section{Removal of the mtcA mutation from each of the $U V$ sensitive strains}

Since the mutations leading to UV sensitivity were induced in strain 302 which had an existing mutation in $m t c A$, conferring sensitivity to MTC, this was removed by incubating each 
Table 2. Transformation frequencies for rifampicin resistance and UV resistance in the wildtype and mutants UVS9, UVS25 and UVS78 of D. radiodurans incubated with DNA isolated from each of the strains

Rifampicin resistance and UV resistance were tested $5 \mathrm{~h}$ and $9 \mathrm{~h}$, respectively, after transformation.

\begin{tabular}{|c|c|c|c|c|}
\hline \multirow[b]{2}{*}{ Donor DNA } & \multicolumn{4}{|c|}{ Recipient: } \\
\hline & Wild-type & UVS9 & UVS25 & UVS78 \\
\hline \multicolumn{5}{|c|}{ (a) Rifampicin resistance } \\
\hline Wild-type & $5.0 \times 10^{-3}$ & $7 \cdot 7 \times 10^{-5}$ & $1.5 \times 10^{-4}$ & $1.6 \times 10^{-4}$ \\
\hline UVS9 & $2.5 \times 10^{-3}$ & $3 \cdot 2 \times 10^{-5}$ & $7.9 \times 10^{-6}$ & $7 \cdot 1 \times 10^{-4}$ \\
\hline UVS25 & $1.3 \times 10^{-3}$ & $1.7 \times 10^{-4}$ & $8.3 \times 10^{-6}$ & $1.7 \times 10^{-4}$ \\
\hline UVS78 & $2.5 \times 10^{-3}$ & $7 \cdot 3 \times 10^{-5}$ & $2 \cdot 3 \times 10^{-5}$ & $2.0 \times 10^{-4}$ \\
\hline \multicolumn{5}{|c|}{ (b) UV resistance } \\
\hline Wild-type & NA & $3.2 \times 10^{-4}$ & $4.0 \times 10^{-5}$ & $2.3 \times 10^{-4}$ \\
\hline UVS9 & NA & $<4.7 \times 10^{-6}$ & $7.5 \times 10^{-5}$ & $1.8 \times 10^{-4}$ \\
\hline UVS25 & NA & $2.4 \times 10^{-4}$ & $5.2 \times 10^{-6}$ & $3.6 \times 10^{-4}$ \\
\hline UVS78 & NA & $3.9 \times 10^{-5}$ & $1 \cdot 1 \times 10^{-4}$ & $<1.7 \times 10^{-7}$ \\
\hline
\end{tabular}

NA, Not applicable.

of the new strains UVS9, 25 and 78, with DNA from the wild-type and selecting for transformants growing on TGY agar containing $0.05 \mu \mathrm{g} \mathrm{MTC} \mathrm{ml}^{-1}$. Transformation frequencies for UVS9, 25 and 78 were $1.0 \times 10^{-2}, 5 \times 10^{-5}$ and $1.0 \times 10^{-2}$, respectively. Two hundred MTCresistant transformants of each of strains 9 and 25 and 347 MTC-resistant transformants of strain 78 were tested for their sensitivity to UV radiation by irradiating streaks of them on TGY agar with $500 \mathrm{~J} \mathrm{~m}^{-2}$. All were resistant to UV radiation. Transformants of UVS9, UVS25 and UVS78 obtained in this way and having the MTC- and UV-resistant phenotype were given the strain description of 91,251 and 781 , respectively.

Isolation of UV-resistant transformants of strains UVS9, 25 and 78 and their phenotype with respect to mitomycin

Strains UVS9, 25 and 78 were incubated with DNA from the wild-type and UV-resistant transformants isolated. These were then tested for resistance or sensitivity to MTC by streak

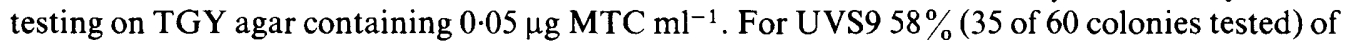
the UV resistant transformant colonies were also MTC-resistant, for UVS25, 56\% (56 of 100) and for UVS78, $54 \%$ (159 of 295). As a control UVS78 was incubated with DNA from strain 301 and UV-resistant transformants were isolated. All were MTC sensitive since the $m t c A$ mutation is common to both UVS78 and 301 and the wild-type allele cannot be substituted for the mutant one. Six UV-resistant, MTC-sensitive transformants of UVS78 were tested for resistance or sensitivity to alkylation-induced mutagenesis. All were sensitive. Six out of six UV-resistant, MTC-resistant clones were resistant to such mutagenesis.

To check the phenotypic effect of each of the mutations $u v s C$, uvsD and uvsE in a wild-type background UV survival curves were obtained for UV-resistant transformants of UVS9, 25 and 78 that were also MTC-resistant and resistant to mutation by alkylating agents by restoration of the wild-type allele at $m t c A$ (Fig. 2). Six of each type were tested. Although there was variation in UV resistance in each group of six, the six transformants of UVS25 were more resistant than the transformants of UVS9 which in turn were more resistant than those of UVS78. The survival curve plotted from the average values for the six UVS9 transformants was identical to that of the wild-type.

\section{Excision of pyrimidine dimers}

The UV-resistant wild-type and strain 302 excised $50 \%$ of the pyrimidine dimers induced by $360 \mathrm{~J} \mathrm{~m}^{-2} \mathrm{UV}$ radiation in about $18 \mathrm{~min}$ while the UV-sensitive double mutants UVS9, UVS25 


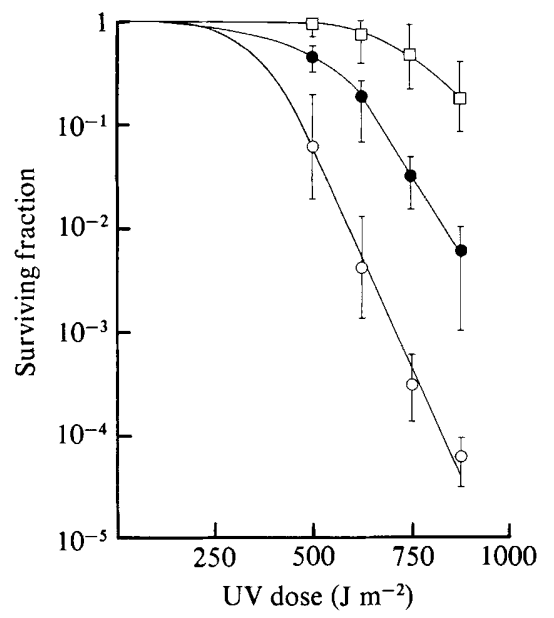

Fig. 2. UV irradiation survival curves of six clones each of strains UVS9, UVS25 and UVS78 transformed with DNA of the wild-type and selected for UV and MTC resistance. These strains were designated $91(\bigcirc), 251(\square)$ and $781(\bigcirc)$, respectively. The average values for each group of six are plotted, the bars representing the range within which individuals in each group fell.

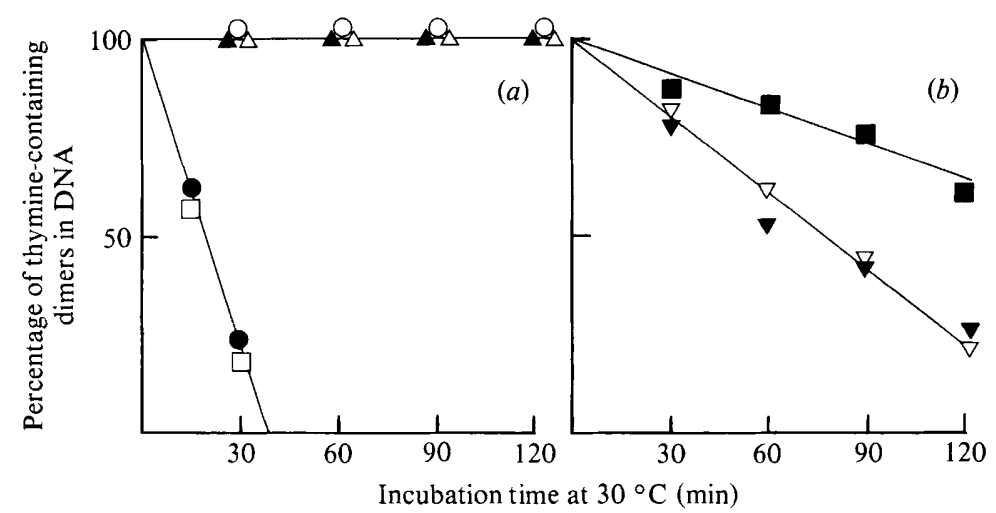

Fig. 3. Rate of loss of thymine containing pyrimidine dimers from the DNA of strains of Deinococcus radiodurans irradiated with $360 \mathrm{~J} \mathrm{~m}^{-2} \mathrm{UV}$ radiation and incubated in TGY broth $(a)$ wild-type (O), the single mutant, $302(\square)$, and the double mutants UVS9 $(\triangle)$, UVS25 $(\mathbf{A})$ and UVS78 $(\bigcirc) ;(b)$ the single mutants $91(\nabla), 251(\nabla)$ and 781

and UVS78 were unable to excise any dimers during $120 \mathrm{~min}$ incubation (Fig. $3 a$ ). The UVresistant single mutants 91,251 and 781 excised dimers, but more slowly than the wild-type, 91 and 251 releasing $50 \%$ in about $80 \mathrm{~min}$ while 781 had released only about $35 \%$ at $120 \mathrm{~min}$ (Fig. $3 b)$.

\section{UV non-mutability of excision-defective strains}

The frequencies of rifampicin-resistant mutants in populations of bacteria grown from UVand non-irradiated suspensions of UVS9, 25 and 78 were calculated from experimental data. The doses of UV administered were $63 \mathrm{~J} \mathrm{~m}^{-2}$ for UVS9 and UVS78 and $126 \mathrm{~J} \mathrm{~m}^{-2}$ for UVS25. For UVS9, the frequency of rifampicin-resistant mutants from an unirradiated population was 4 $\times 10^{-7}$ and in an irradiated one, $6 \times 10^{-7}$. For UVS25, the corresponding values were $8 \times 10^{-7}$ and $6 \times 10^{-7}$ and for UVS78 they were $2 \times 10^{-6}$ and $1.2 \times 10^{-6}$. Thus there was no evidence for $\mathrm{UV}$-induced mutation. 
Table 3. Summary of results for UVS78

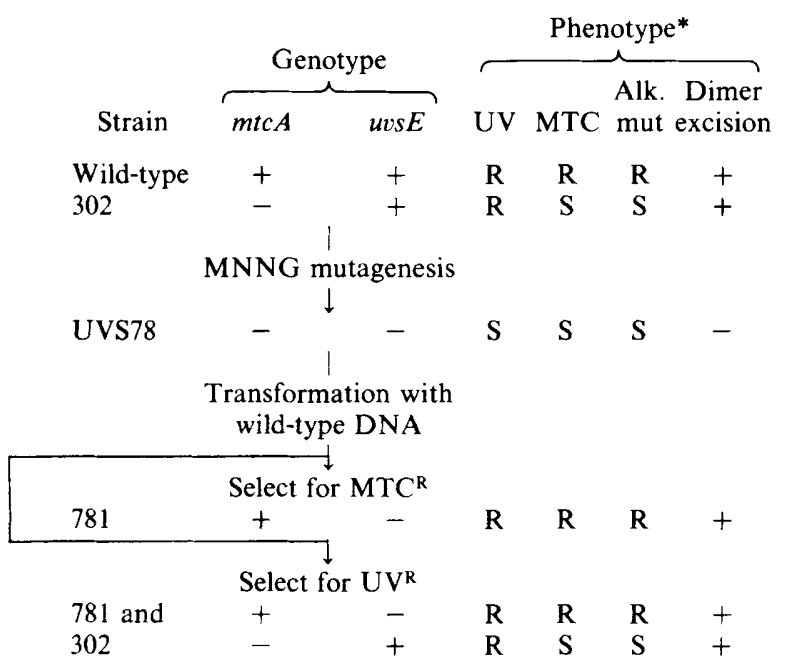

* Alk. mut denotes alkylation mutagenesis; $\mathrm{R}$ denotes resistant and $\mathrm{S}$ denotes sensitive.

The results for the isolation of UVS78 and its transformation to other genotypes are summarized in Table 3 . The results for UVS9 and 25 were similar.

\section{DISCUSSION}

In order to increase the chance of isolating excision-defective mutants of $D$. radiodurans, it was decided to mutagenize a population of strain 302 rather than wild-type because the former yields fifty times as many MNNG-induced mutants as the latter. It was assumed that excisiondefective strains would be generated by single mutations in genes analogous to the $u v r A, B$ and $C$ genes of Escherichia coli. It was also assumed that the $m t c A$ gene which had been demonstrated to have a role in the resistance of the wild-type to alkylation mutagenesis and MTC-induced lethality did not contribute to UV resistance since a strain mutant in this gene has the same UV resistance as the wild-type. This implied that the mutant $m t c A$ gene could be replaced by its wildtype allele in putative excision defective strains in order to study the effect of any newly induced mutation.

Three strains were isolated having some of the properties of excision-deficient mutants, viz. UV sensitivity and MMS resistance, and were subsequently shown to be unable to excise UVinduced thymine-containing pyrimidine dimers. The three newly induced mutations in the three strains UVS9, 25 and 78 were different from each other on the basis that DNA from each of the strains could transform both of the others to UV resistance and so the respective genes were designated uvs $C, D$ and $E$. The genes uvs $A$ and $B$ have already been assigned, mutations in these genes causing sensitivity to UV radiation in strains 303 and 263, respectively (Moseley \& Copland, 1978). However, when the mutant $m t c A$ gene in each of UVS9, 25 and 78 was replaced by its wild-type allele then strains possessing only one of the mutations uvs $C, D$ or $E$ regained resistance to UV radiation, i.e. mutations in $u v s C, D$ or $E$ could only confer UV sensitivity in strains already mutant in $m t c A$ or vice versa. Thus the wild-type genes $u v s C, D$ or $E$ conferred UV resistance on a strain in the presence of a mutant $m t c A$ gene (strain 302) and conversely a wild-type $m t c A$ gene conferred UV resistance on strains mutant in $u v s C, D$ or $E$ (strains 91, 251 and 781, respectively). The UV-resistant strains 91,251 and 781 carrying single mutations in $u v s C, D$ and $E$, respectively, excised thymine-containing pyrimidine dimers, but at varying rates and in all cases slower than that of the wild-type and 302. The lower rates in these mutants is 
taken as a reflection of the relative importance of the $u v s C, D, E$ pathway compared with that governed by the $m t c A$ gene, viz. the $u v s C, D, E$ pathway excises dimers at a faster rate than does the $m t c A$ pathway and since on its own it excises pyrimidine dimers at the same rate as when both pathways are operating must play a major role in the wild-type. However, a maximum rate of dimer excision does not necessarily give maximum survival since the strains mutant in $u v s D$ (UVS25) had slower excision rates than wild-type, but a greater resistance to UV radiation.

The evidence for two excision pathways offers an explanation for the prior lack of success in isolating excision-deficient mutants since it is now clear that two independent mutations are required for the UV-sensitive phenotype to be displayed, a result fortuitously gained by choosing to treat with MNNG a strain already mutant in one of them. The possession by an organism of two excision pathways either one of which is capable of conferring wild-type resistance to UV radiation has not been reported previously. Two UV endonucleases, I and II, have been isolated from wild-type Micrococcus luteus (Riazuddin \& Grossman, 1977a, b), but both are apparently required in a cell to confer UV resistance (Riazuddin et al., 1977). Thus a strain of $M$. luteus lacking both endonucleases and one lacking only endonuclease I were UV-sensitive while a strain lacking only endonuclease II could not be isolated by transformation with wild-type DNA of the strain lacking both. If the presence of endonuclease I alone had conferred UV resistance its selection would have been straightforward.

None of the mutations $u v s C, D$ or $E$ has been shown to be closely linked to $m t c A$ since UV resistant transformants selected from the double mutants UVS9, 25 and 78 incubated with wildtype DNA fell approximately equally into two groups, viz. those which were MTC-sensitive, presumably having the mutant genes $u v s C, D$ or $E$ replaced by the wild-type allele, giving strains isogenic to 302 , and those which were resistant to MTC and alkylation mutagenesis, presumably having the mutant $m t c A$ gene replaced by its wild-type allele, but containing singly the mutant genes uvs $C, D$ or $E$ (strains 91,251 and 781, respectively). Thus, for example, in UVS9 about $50 \%$ of the transformation events replaced the $m t c A$ gene and $50 \%$ the $u v s C$ gene and there was no marked skew to the UV-resistant MTC-resistant phenotype which one would expect if a substantial number of single transformation events replaced both genes. The values of 58,56 and $54 \%$ for the proportion of transformants of UVS9, 25 and 78 having the UV-resistant MTCresistant phenotype are assumed not to be significantly different from $50 \%$ in view of the numbers of clones screened.

Excision-deficient mutants of $E$. coli are much more mutable by UV light than the wild-type (Hill, 1965) and Witkin (1969) calculated that an unexcised dimer must be at least 500 times more likely to cause a mutation than an excised one, as a result of its passing along an errorprone repair pathway. Deinococcus radiodurans has been reported not to possess an error prone repair pathway for UV-induced damage (Sweet \& Moseley, 1974, 1976) and this is confirmed by the lack of any induced mutation in excision-deficient mutants of $D$. radiodurans.

This investigation was supported by a grant from the Medical Research Council.

\section{REFERENCES}

Anderson, A. W., Nordan, H. C., Cain, R. F., Parrish, G. \& Duggan, D. (1956). Studies on a radio-resistant micrococcus. I. Isolation, morphology, cultural characteristics and resistance to gamma radiation. Food Technology 10, 575-578.

Boling, M. E. \& Setlow, J. K. (1966). The resistance of Micrococcus radiodurans to ultraviolet radiation. III. A repair mechanism. Biochimica et biophysica acta 123, 26-33.

Brooks, B. W. \& Murray, R. G. E. (1981). Nomenclature for "Micrococcus radiodurans" and other radiation resistant cocci: Deinococcaceae fam. nov. and Deinococcus gen. nov, including five species. International Journal of Systematic Bacteriology 31, 353-360.
Fox, G. E., Stackebrandt, E., Hespell, R. B., Gibson, J. Maniloff, J., Dyer, T., Wolfe, R. S., Balch, W., TANner, R., Magrum, L., Zablen, L. B., Blakemore, R., Gupta, R., Luehrsen, K. R., Bonen, L., Lewis, B. J., Chen, K. N. \& Woese, C. R. (1980). The phylogeny of prokaryotes. Science 209, 457-463.

HILL, R. F. (1958). A radiation sensitive mutant of Escherichia coli. Biochimica et biophysica acta 30, 636637.

HILL, R. F. (1965). Ultraviolet-induced lethality and reversion to prototrophy in Escherichia coli strains with normal and reduced dark repair ability. Photochemistry and Photobiology 4, 563-568.

Howard-Flanders, P. \& Theriot, L. (1962). A 
method for selecting radiation sensitive mutants of Escherichia coli. Genetics 47, 1219-1224.

Moseley, B. E. B. (1967). The isolation and some properties of radiation-sensitive mutants of Micrococcus radiodurans. Journal of General Microbiology 49, 293-300.

Moseley, B. E. B. (1983). Photobiology and radiobiology of Micrococcus (Deinococcus) radiodurans. Photochemical and Photobiological Reviews 7, 223274.

Moseley, B. E. B. \& Copland, H. J. R. (1975). Isolation and properties of a recombination-deficient mutant of Micrococcus radiodurans. Journal of Bacteriology 121, 422-428.

Moseley, B. E. B. \& Copland, H. J. R. (1978). Four mutants of Micrococcus radiodurans defective in the ability to repair DNA damaged by mitomycin C, two of which have wild-type resistance to ultraviolet radiation. Molecular and General Genetics 160, 331337.

Moseley, B. E. B., Mattingly, A. \& Copland, H. J. R. (1972). Sensitization to radiation by loss of recombination ability in a temperature-sensitive DNA mutant of Micrococcus radiodurans held at its restrictive temperature. Journal of General Microbiology 72, 329-338.

Riazuddin, S. \& Grossman, L. (1977a). Micrococcus luteus correndonucleases. I. Resolution and purification of two endonucleases specific for DNA containing pyrimidine dimers. Journal of Biological Chemistry 252, 6280-6286.

Riazuddin, S. \& Grossman, L. (1977b). Micrococcus luteus correndonucleases. II. Mechanisms of action of two endonucleases specific for DNA containing pyrimidine dimers. Journal of Biological Chemistry 252, 6287-6293.

Riazuddin, S., Grossman, L. \& Mahler, I. (1977). Micrococcus luteus correndonucleases. III. Evidence for involvement in repair in vivo of two endonucleases specific for DNA containing pyrimidine dimers. Journal of Biological Chemistry 252, 62946298.

Sweet, D. M. \& Moseley, B. E. B. (1974). Accurate repair of ultraviolet induced damage in Micrococcus radiodurans. Mutation Research 23, 311-318.

Sweet, D. M. \& Moseley, B. E. B. (1976). The resistance of Micrococcus radiodurans to killing and mutation by agents which damage DNA. Mutation Research 34, 175-186.

Tempest, P. R. \& Moseley, B. E. B. (1978). Role of the gene $m t c A$ in the resistance of Micrococcus radiodurans to the lethal effects of mitomycin $\mathrm{C}$ and alkylation mutagenesis. In DNA Repair Mechanisms, pp. 283-286. Edited by P. C. Hanawalt, E. C. Friedberg \& C. F. Fox, New York: Academic Press.

Tempest, P. R. \& Moseley, B. E. B. (1980). Defective excision repair in a mutant of Micrococcus radiodurans hypermutable by some monofunctional alkylating agents. Molecular and General Genetics 179, 191199.

Tirgari, S. \& Moseley, B. E. B. (1980). Transformation in Micrococcus radiodurans: measurements of various parameters and evidence for multiple, independently segregating genomes per cell. Journal of General Microbiology 119, 287-296.

WiTKIN, E. M. (1969). The mutability toward ultraviolet light of recombination-deficient strains of Escherichia coli. Mutation Research 8, 9-14. 